\title{
BUMETANIDE-ENHANCED PHENOBARBITAL IN NEONATAL SEIZURE MODEL
}

Anticonvulsant efficacy of phenobarbital, bumetanide, and a combination of these drugs was studied in an in vitro hippocampal rat pup (4-7 days) preparation, with recurrent seizures induced by exposure to low-Mg solution, at the Department of Neurology, Massachusetts General Hospital, Boston. The combination of phenobarbital and bumetanide abolished these experimental seizures in $70 \%$ of hippocampi and reduced their frequency and duration in the remaining 30\%. The GABA-mediated outward flow of $\mathrm{Cl}$ in immature neurons is excitatory, contributing to a poor response to GABAergic anticonvulsants such as phenobarbital and benzodiazepines. The diuretic bumetanide blocks the $\mathrm{Na} \mathrm{K} \mathrm{Cl}$ transporter system and prevents the outward flow of $\mathrm{Cl}$ ions. Alteration of $\mathrm{Cl}$ ion transport by bumetanide facilitates the anticonvulsant action of phenobarbital in immature animal brain, providing a potential effective therapy for neonatal seizures. (Dzhala VI, Brumback AC, Staley KJ. Bumetanide enhances phenobarbital efficacy in a neonatal seizure model. Ann Neurol February 2008;63:222-235). (Respond: Dr Kevin J Staley, MD, Department of Neurology, Massachusetts General Hospital, 55 Fruit Street, VBK 910, Boston, MA 02114. E-mail: kstaley@partners.org).

COMMENT. The authors propose a clinical trial of bumetanide in combination with phenobarbital in the treatment of neonates with seizures. Previous studies cited have demonstrated a low risk of side effects with bumetanide in sick infants (Sullivan JE et al. 1996). The above laboratory study and others by my colleague, Dr Sookyong Koh, recipient of the Dreiffus-Penry Epilepsy Award at the AAN meeting, April 15, have advanced our understanding of seizure mechanisms and control of epilepsy-associated behavioral changes in the young.

\section{INFECTIOUS DISORDERS}

\section{VARICELLA ZOSTER VIRUS VASCULOPATHIES}

The clinical, CSF, imaging, and virological features of varicella zoster virus (VZV) vasculopathies in 30 patients ( 7 children) are reviewed by researchers at various centers in the US and internationally. The data obtained on the 7 children, ages 1-7 and 18, show rash in 5, CSF pleocytosis in 6, focal lesions on MRI/CT in 7, focal vascular abnormalities by angiography or MRA in 3 of 4 tested, small vessel involvement in 2 and mixed in 5, CSF VZV DNA in 2, and IgG in 5, and reduced serum/CSF ratio of VZV IgG in 5, confirmation of intrathecal synthesis. Average time from rash to neurologic symptoms and signs and virologic analysis was 4.1 months. In children it varied from 1-2 days to 3 months. Detection of anti-VZV IgG antibody in CSF is a more sensitive indicator of VZV vasculopathy than VZV DNA $(\mathrm{p}<0.001)$. Of 15 patients ( 13 adults and 2 children) treated with IV acyclovir alone for 10-28 days, $66 \%$ improved or stabilized compared to $75 \%$ of 12 patients ( 9 adults and 3 children) who received both IV acyclovir and steroids. Of 2 children not treated, 1 stabilized and the other improved slowly. (Nagel MA, Cohrs RJ, Mahalingam R et al. The 